\title{
Antioxidant and neuroprotective effects of lycopene and insulin in the hippocampus of streptozotocin-induced diabetic rats
}

\author{
RANA MALEKIYAN, ALIREZA ABDANIPOUR, DAVOOD SOHRABI and IRAJ JAFARI ANARKOOLI \\ Department of Anatomy, Faculty of Medicine, Zanjan University of Medical Sciences, Zanjan 45139-56111, Iran
}

Received January 22, 2018; Accepted October 24, 2018

DOI: $10.3892 /$ br.2018.1171

\begin{abstract}
In the present study the antioxidant and neuroprotective effects of insulin and lycopene on passive avoidance memory, total antioxidant capacity (TAC), malondialdehyde activity (MDA) and prevention of apoptosis in the hippocampus streptozotocin-induced diabetic rats were examined. The rats were randomly divided to six experimental groups ( $n=8$ per group): Non-diabetic (controls); diabetic; diabetic treated with lycopene; diabetic treated with insulin; diabetic treated with lycopene and insulin; and normal treated with lycopene. Intraperitoneal injection of single dose $(60 \mathrm{mg} / \mathrm{kg})$ streptozotocin (STZ) was used to induce the diabetes rat model. The shuttle box test was used for learning and memory assessment. Rats were then sacrificed and hippocampi tissue isolated from the two hemispheres to determine TAC and MDA. Apoptosis rate was also evaluated by terminal deoxynucleotidyl transferase dUTP nick-end labeling and acridine orange staining assays. The results indicated that lycopene and insulin, solely or in combination, prevented hippocampal neuronal cell death and improved learning and cognition by increasing TAC and decreasing MDA. Collectively, the findings presented herein suggest that insulin and lycopene co-treatment has neuroprotective effect, and ameliorates STZ-induced learning and memory impairment and apoptotic cell death in the hippocampal regions of diabetic rats.
\end{abstract}

\section{Introduction}

Diabetes mellitus (DM) is a chronic metabolic disorder in which the pancreas produces insufficient or no insulin to match the body's demands (type $1 \mathrm{DM}$ ), or in which the body is unable to effectively utilize the insulin produced

Correspondence to: Dr Iraj Jafari Anarkooli, Department of Anatomy, Faculty of Medicine, Zanjan University of Medical Sciences, Mahdavi Blvd, Shahrak-e Karmandan, Zanjan 45139-56111, Iran

E-mail: anarkool@zums.ac.ir

Key words: lycopene, diabetes, insulin, hippocampus, apoptosis, rat (type 2 DM) (1). The prevalence of diabetes is increasing to the extent that the number of adults with diabetes has been estimated to be 592 million by 2035 (2-5). DM is linked with oxidative stress occurring as a consequence of increased formation of free radicals, including superoxide $\left(\mathrm{O} 2^{-}\right)$and hydroxyl $(\mathrm{OH})$ radicals, and decreased activity of antioxidant defense systems (6). Hyperglycemia increases the formation of reactive oxygen species (ROS) via several pathways including glucose autoxidation, the polyol pathway and non-enzymatic protein glycation (7). Free radicals can adversely affect important biomolecules including carbohydrates, proteins and DNA (8). Oxidative damage to various brain regions results in long-term complications, morphological abnormalities and memory impairments (9). Emerging evidence suggests that oxidative damage associated with DM may negatively impact the central nervous system (CNS), causing cognitive impairment in addition to complications in the peripheral nervous system (2). In the CNS, the hippocampus is considered a particular target for diabetes-related changes (10). The hippocampus is a component of the limbic system of the brain and considered as an integration center for cognitive functions including learning and memory (11). Diabetes has been associated with cognitive and memory impairments, indicating that the hippocampus may be affected by the disease (12). Apoptosis may be considered as a pathway for hyperglycemia-induced hippocampal neuronal cell death (13). DNA fragmentation, cell shrinkage and nuclei membrane blebbing are morphological characteristics of apoptosis (14). The neuroprotective effect of antioxidants has been indicated in the treatment of experimental neurodegenerative animal models (5). Consumption of natural antioxidants reportedly reduces the risk of cancer, cardiovascular disease and diabetes, among other diseases (15). Lycopene, which naturally occurs in tomato and other fruits including papaya, pink guava and watermelon, has long been known to have potential health-promoting properties (16). Among naturally-occurring carotenoids, lycopene appears to best scavenge free radicals (17). Lycopene may also penetrate the blood-brain barrier and prevail in the CNS (18). The purpose of the present study was to investigate the effect of lycopene, insulin and their co-treatment in preventing apoptosis, on the levels of total antioxidant capacity (TAC) and malondialdehyde activity (MDA), within the hippocampus of streptozotocin (STZ)-induced diabetic rats. 


\section{Materials and methods}

Animals and study design. A total of 48 adult male Wistar rats (weighing 200-250 g, aged 8-10 weeks old) were purchased from the Baqiyatallah University of Medical Sciences, Tehran, Iran. The animals were housed under standard laboratory conditions (12-h light/dark cycle, room temperature of $21-22^{\circ} \mathrm{C}$ and $45-55 \%$ humidity) with ad libitum access to food and tap water. The experimental protocols were reviewed and approved by the Institutional Animal Ethics Committee of Zanjan University of Medical Sciences (ZUMS), Zanjan, Iran (approval no. ZUMS.REC.1394.29), and conducted in accordance with the ethical guidelines approved by the Institutional Animal Ethics Committee of ZUMS (IAEC no. 03/028/07). An overnight fast was followed by a single dose of $60 \mathrm{mg} / \mathrm{kg}$ STZ (Sigma-Aldrich; Merck KGaA, Darmstadt, Germany) prepared in citrate buffer ( $\mathrm{pH} 4.4 ; 0.1 \mathrm{M})$, injected intraperitoneally (i.p.) to induce diabetes (19). Blood samples $(0.2-0.5 \mathrm{ml})$ were collected through the tail vein, and glucose levels measured using a glucometer (Accu-Chek; Roche Diagnostics GmbH, Mannheim, Germany); diabetes was confirmed $72 \mathrm{~h}$ after STZ injection. Animals with fasting blood-glucose levels $>250 \mathrm{mg} / \mathrm{dl}$ (20) were selected and used for the present study. Body weights and blood-glucose levels were determined prior to the experiment and at the end of the experiment to evaluate the effects of lycopene and insulin. Rats were randomly divided into six groups, with each group consisting of 8 animals. The first group consisted of non-diabetic control animals. The second group served as the control group treated with lycopene $[4 \mathrm{mg} / \mathrm{kg} / \mathrm{day}$ per os (p.o.); lycopene was dissolved in double distilled water following trituration with 5\% Tween-80] (21). The third and fourth groups were the diabetic control and diabetic animals treated with lycopene ( $4 \mathrm{mg} / \mathrm{kg} /$ day p.o.), respectively. The fifth group was the diabetic group treated with insulin (1 to 2 units per day, i.p.), and the sixth group comprised of diabetic animals administered lycopene $(4 \mathrm{mg} / \mathrm{kg}$ p.o.) and insulin (1-2 units a day, i.p.) simultaneously. On the third day of administration, the control and diabetic control groups received $0.5 \mathrm{ml}$ normal saline while the other groups continued to receive insulin and/or lycopene. Drug administration continued for 8 weeks and at the end of the eighth week, learning and memory were evaluated for two days using the shuttle box test. The animals under deep anesthesia were sacrificed; blood samples $(0.2-0.5 \mathrm{ml})$ were collected through the tail vein, and blood-glucose levels were measured. The hippocampi were isolated following rapid removal of the brains. To perform a terminal deoxynucleotidyl transferase dUTP nick-end labeling (TUNEL) assay and acridine orange (AO) staining, left hippocampi were fixed in $10 \%$ formalin, and right hippocampi were stored in cryotubes at $-70^{\circ} \mathrm{C}$ in order to assess TAC and MDA.

Passive avoidance task. The behavioral experiments were conducted using a two-way shuttle box system (Borj Sanaat Co., Tehran, Iran). The apparatus and procedure were as described previously (22). In brief, the step-through passive avoidance apparatus consisted of a light chamber $\left(27 \times 14.5 \times 14 \mathrm{~cm}^{3}\right)$ made of transparent plastic and a dark chamber $\left(27 \times 14.5 \times 14 \mathrm{~cm}^{3}\right)$ made of dark opaque plastic. The floors of both chambers were made of stainless steel rods (3-mm diameter) spaced $1 \mathrm{~cm}$ apart. The floor of the dark chamber could be electrified using a shock generator. A rectangular opening $\left(6 \times 8 \mathrm{~cm}^{2}\right)$ was located between the two chambers and could be closed by an opaque guillotine door.

First, all experimental groups received two trials to habituate and acclimatize the rats to the apparatus. For these trials, the rats were placed in the light compartment of the apparatus facing away from the door, and $10 \mathrm{sec}$ later, the guillotine door was raised. Upon the rat entering the dark compartment, the door was closed, and after $30 \mathrm{sec}$, the rats were taken from the dark compartment and placed in their home cage. The habituation trial was repeated after $30 \mathrm{~min}$ and followed after the same interval by the first acquisition trial. The entrance latency to the dark compartment (step through latency, STLa) was recorded when the animal had placed all four paws in the dark compartment. For the training of the animals, as soon as they had spontaneously entered into the dark compartment, the guillotine door was lowered, and a mild electrical shock $(0.5 \mathrm{~mA})$ was applied for $3 \mathrm{sec}$; after $30 \mathrm{sec}$, the rat was returned to its home cage. Then, after $2 \mathrm{~min}$, the procedure was repeated. The rat received a foot-shock each time it reentered the dark compartment with all four paws placed in the compartment; training was terminated when the rat remained in the light compartment for $120 \mathrm{sec}$. The number of trials (entries into the dark chamber) were recorded. Long-term memory was tested within $24 \mathrm{~h}$ after the passive avoidance learning (PAL) acquisition trial. The rats were placed in the lighted chamber as in PAL training session, and $10 \mathrm{sec}$ later, the guillotine door was raised, and the step-through latency (STLr) and the time spent in the dark compartment (TDC) were recorded for up to $600 \mathrm{sec}$. If the rat did not enter the dark compartment within $600 \mathrm{sec}$, the retention test was terminated, and a ceiling score of $600 \mathrm{sec}$ was assigned. During this session, the electric shocks were not applied to the grid floor.

Tissue preparation. The hippocampus samples obtained from rats were immersed in $10 \%$ formalin for $72 \mathrm{~h}$ to allow fixation at room temperature. A slow step-wise dehydration was performed for tissue processing by adding a series of ethanol solutions (50-100\%) of increasing concentration. Samples were washed with xylene prior to the addition of each new alcohol solution. Following dehydration, the hippocampal samples were embedded in melt paraffin. Sectioning was performed using a rotary microtome, from which slices of $5 \mu \mathrm{m}$ thickness were obtained. The slices were mounted on microscope slides and any paraffin adhering to the mounted sections was dissolved by passive clearance with chloroform.

TUNEL assay. In-situ DNA fragmentation was visualized by the TUNEL method according to a previous study (23) using an in situ cell death detection POD kit (Roche Diagnostics $\mathrm{GmbH}$ ) (24). Briefly, dewaxed tissue sections were predigested with proteinase $\mathrm{K}(20 \mathrm{mg} / \mathrm{ml}$ in $10 \mathrm{mmol} / 1$ Tris- $\mathrm{HCl}$, pH 7.6) for $30 \mathrm{~min}$ at room temperature, then rinsed three times with phosphate-buffered saline solution (PBS). Subsequently, treatment with $3 \% \mathrm{H}_{2} \mathrm{O}_{2}$ for $10 \mathrm{~min}$ at room temperature was used to block endogenous peroxidase activity, and the sections were incubated with the TUNEL reaction mixture for 
$60 \mathrm{~min}$ at $37^{\circ} \mathrm{C}$. The slides were then rinsed three times with PBS and incubated with convertor peroxidase for $30 \mathrm{~min}$ in a humidified chamber at room temperature. Following three washes with PBS, diaminobenzidine chromogenic substrate (Roche Diagnostics $\mathrm{GmbH}$ ) was added to sections for $10 \mathrm{~min}$ at room temperature. The sections were counterstained with hematoxylin for $30 \mathrm{sec}$ at room temperature. The number of TUNEL-positive cells in the hippocampal region were counted under a light microscope (Olympus BX51; Olympus Corporation, Tokyo, Japan) by double-blinded observation and analyzed with Digital Imaging Solution Cell Software version 1.1 (Olympus Soft Imaging Solutions GmbH, Münster, Germany).

AO staining. $\mathrm{AO}$ is a metachromatic fluorescence probe which is used to demonstrate the degree of nuclear DNA susceptibility to diabetes-induced denaturation by distinguishing between native double-stranded DNA (green fluorescence) and denatured single-stranded DNA (red fluorescence) (5). Samples were washed with distilled water (5 min) and PBS (5 min) and then stained with freshly prepared AO $(0.19 \mathrm{mg} / \mathrm{ml}$ in McIlvain phosphate-citrate buffer, $\mathrm{pH}$ 4.0) for $20 \mathrm{~min}$, and finally counterstained with hematoxylin for $5 \mathrm{~min}$, all at room temperature. To measure the percentage of dead cells, samples were assessed on the same day using a fluorescent microscope (Olympus BX51). A total of 8 samples from each group were selected randomly. For each sample, 5 sections, and in each section, 5 regions [CA1, CA2, CA3, CA4 and dentate gyrus (DG)], were evaluated at random. The total number of cells and the number of dead cells were determined in each region (23).

$T A C$. The hippocampus samples obtained from rats were lysed by adding $1 \mathrm{ml}$ lysis buffer $\left(154 \mathrm{mM} \mathrm{NH}_{4} \mathrm{Cl}, 10 \mathrm{mM} \mathrm{KHCO}_{3}\right.$, 0.1 mM EDTA, pH 7.4). Samples were then homogenized with a sterile syringe and homogenizer. The samples were centrifuged at 7,000 x $\mathrm{g}$ for $20 \mathrm{~min}$ at room temperature, and the supernatants were collected and aliquoted. Samples were stored at $-20^{\circ} \mathrm{C}$ prior to assessment of TAC and MDA. The TAC assay was performed using a TAC kit (Abcam, Cambridge, MA, USA) and the method described by Prieto et al (25). Different concentrations of extracts $(0.50,0.75,1.00$ and $1.50 \mathrm{mg} / \mathrm{ml})$ and ascorbic acid as standard were added to $3 \mathrm{ml}$ reagent solution $\left(0.6 \mathrm{M} \mathrm{H}_{2} \mathrm{SO}_{4}, 28 \mathrm{mM} \mathrm{Na}_{2} \mathrm{HPO}_{4}\right.$ and $4 \mathrm{mM}$ ammonium molybdate). The samples were incubated for $90 \mathrm{~min}$ at $25^{\circ} \mathrm{C}$ following shaking, and centrifuged at $700 \mathrm{x}$ g for $10 \mathrm{~min}$ at room temperature. The absorbance of the supernatant was then determined at $490 \mathrm{~nm}$ with an ELISA plate reader. Distilled water $(1 \mathrm{ml})$ was used as blank, processed in the same way. TAC was expressed as ascorbic acid equivalent per dry weight of extract.

MDA assay. MDA content, as a measure of lipid peroxidation, was assayed in the form of thiobarbituric acid-reactive substances (TBARS) by the method of Wills (26) using a lipid peroxidation MDA assay kit (Abcam). Briefly, $0.5 \mathrm{ml}$ post mitochondrial supernatant and $0.5 \mathrm{ml}$ Tris $\mathrm{HCl}$ were incubated at $37^{\circ} \mathrm{C}$ for $2 \mathrm{~h}$. Following incubation, $1 \mathrm{ml} \mathrm{10 \%}$ trichloroacetic acid was added and centrifuged at $1,000 \mathrm{x}$ g for $10 \mathrm{~min}$ at room temperature. To $1 \mathrm{ml}$ of supernatant, $1 \mathrm{ml} 0.67 \%$ thiobarbituric acid was added and the tubes were retained in boiling water for $10 \mathrm{~min}$. Following cooling, $1 \mathrm{ml}$ double distilled water was added and absorbance was measured at $535 \mathrm{~nm}$ with an ELISA plate reader. TBARS were quantified using an extinction coefficient of $1.56 \times 10^{5} \mathrm{M}^{-1} \mathrm{~cm}^{-1}$. Tissue protein was estimated using the Biuret method and the brain malondialdehyde content expressed as nmol malondialdehyde/mg protein (27).

Statistical analysis. For data analysis, SPSS 15.0 software (SPSS, Inc., Chicago, IL, USA) was used. All data are presented as the mean \pm standard error of the mean. To compare differences between the means of multiple groups, one-way analysis of variance followed by Tukey's post hoc test was used. $\mathrm{P}<0.05$ was considered to indicate statistical significance.

\section{Results}

Step-through latency in the acquisition trial. On assessing delay in the acquisition phase (step-through to the dark compartment), there were no significant differences between the groups (Fig. 1A).

Responding at the end of trial to acquisition (TA). At $24 \mathrm{~h}$ after training, memory assessment showed that TA for step-through between the two chambers was significantly increased in the diabetic group without treatment compared with in the control group $(\mathrm{P}<0.05)$, indicating that the induction of diabetes could increase TA. Comparing the diabetic group without treatment with the groups treated with insulin and lycopene individually and simultaneously, the increase in TA observed was statistically significant $(\mathrm{P}<0.05$; Fig. $1 \mathrm{~B})$.

Step-through latency (STLr). At $24 \mathrm{~h}$ after training, STLr of the diabetic group without treatment was significantly decreased compared with that of the control group $(\mathrm{P}<0.05)$, Furthermore, in the diabetic group without treatment, STLr was significantly reduced compared with in the diabetic groups treated with insulin, lycopene or both compounds simultaneously $(\mathrm{P}<0.05$; Fig. 1C).

Time spent in dark compartment (TDC). Comparisons of total time spent in the dark compartment of the shuttle box between diabetic and control rats revealed significant differences among groups. In the diabetic group without treatment, probably owing to increased TA between the two chambers, and thereby reduced STLr, TDC was significantly increased compared with in the control group $(\mathrm{P}<0.05)$. In the diabetic groups treated with insulin, lycopene or both compounds simultaneously, TA between the two chambers was reduced compared with in the diabetic group without treatment, resulting in a significant reduction in TDC in these groups, compared with in the diabetic group without treatment $(\mathrm{P}<0.05$; Fig. 1C).

TAC in the hippocampus. The TAC of blood in the diabetic group was significantly lower than that in the control group $(\mathrm{P}<0.05)$. By contrast, the diabetic rats that underwent treatment for 8 weeks with insulin, lycopene and a combination thereof exhibited significant increases in TAC compared with the diabetic rats without treatment $(\mathrm{P}<0.05$; Fig. 2$)$. 

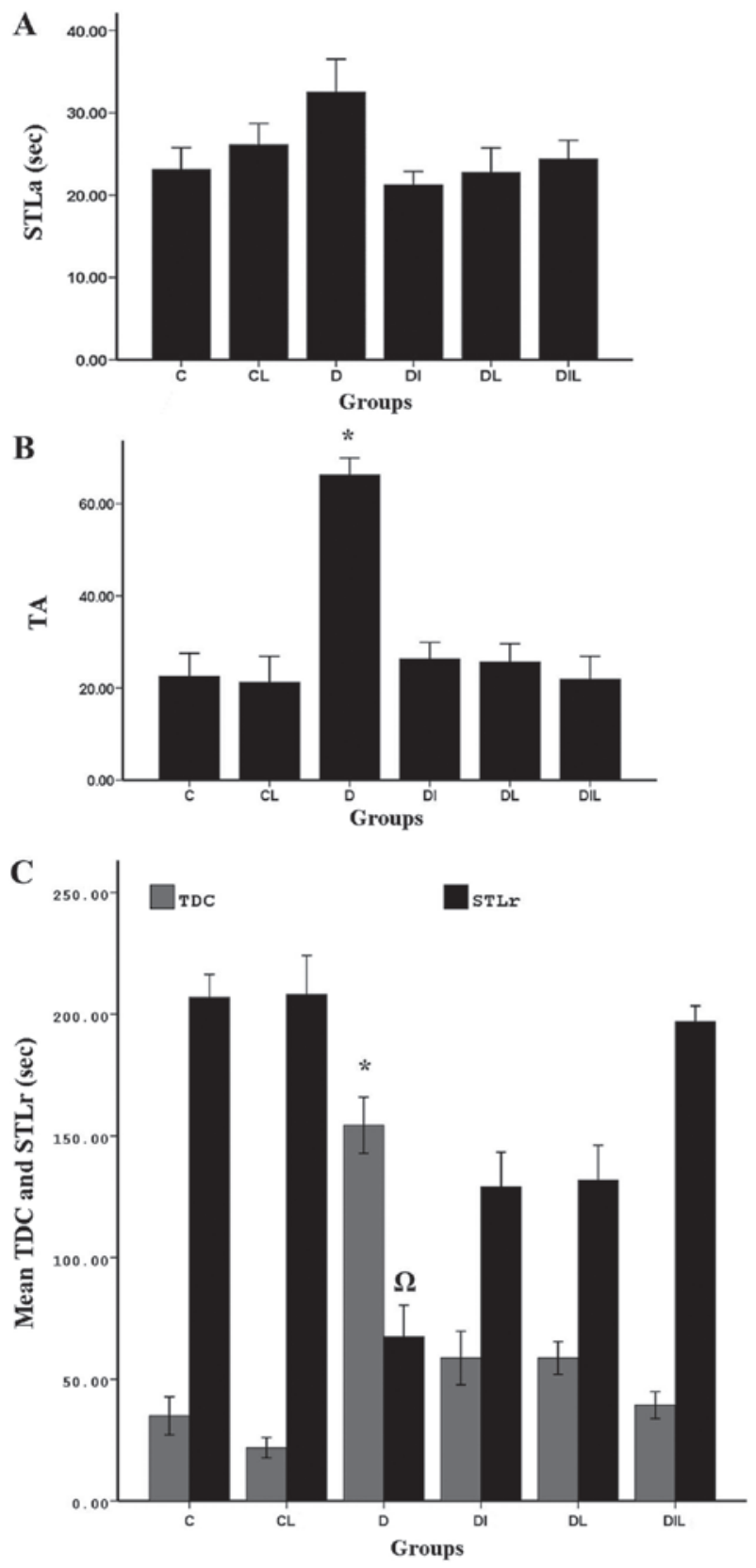

Figure 1. Effect of lycopene and insulin on (A) STLa, (B) trial to acquisition (TA) and (C) STLr and TDC in diabetic rats subjected to the passive avoidance learning test. " $\mathrm{P}<0.05$, group $\mathrm{D}$ vs. other experimental groups; ${ }^{\Omega} \mathrm{P}<0.05$, group D vs. other experimental groups. The bars indicate the mean \pm standard error of the mean. C, control; CL, control group treated with lycopene; D, untreated diabetic group; DI, diabetic group treated with insulin; DL, diabetic group treated with lycopene; DIL, diabetic group treated with insulin and lycopene; STLa, step-through latency in the acquisition trial; STLr, step-through latency; TDC, time spent in dark compartment.

MDA in the hippocampus. MDA was significantly increased in the diabetic group compared with in the control group $(\mathrm{P}<0.05)$. In the diabetic rats treated for 8 weeks with insulin, lycopene or a combination thereof, MDA was significantly lower than that in the diabetic rats without treatment $(\mathrm{P}<0.05$; Fig. 2).

AO staining results. AO is a nucleic acid-selective metachromatic stain useful for cell cycle determination. In this staining assay, the dead and intact cells were red and

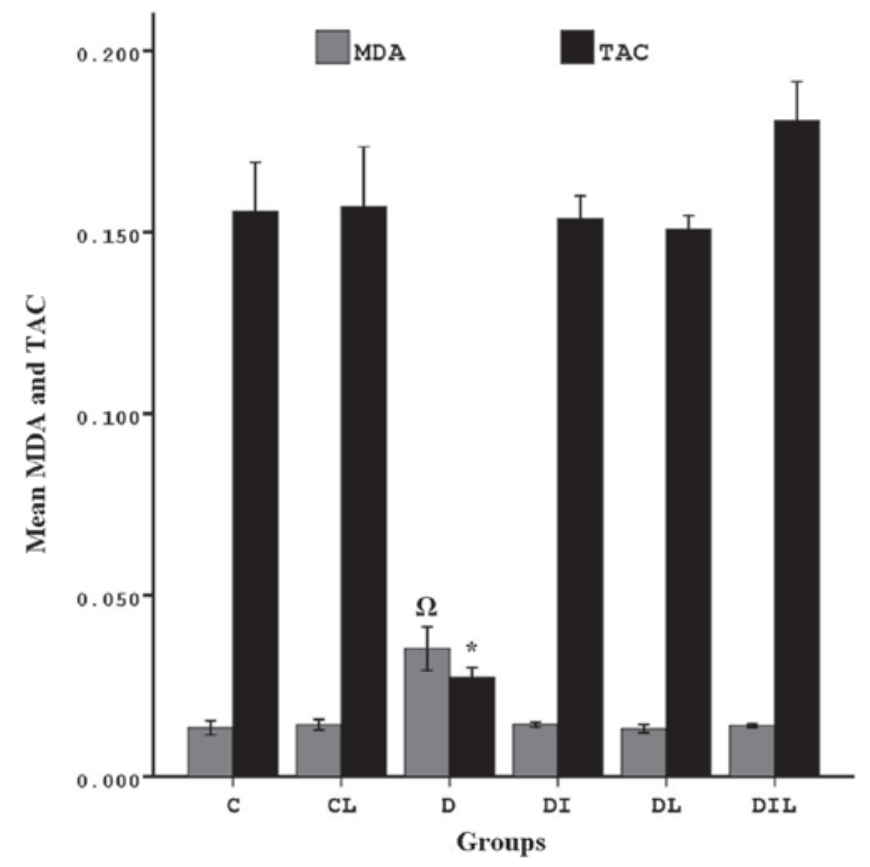

Figure 2. Effect of lycopene and insulin on TAC and MDA in the hippocampus of diabetic rats. MDA: ${ }^{\Omega} \mathrm{P}<0.05$, group D vs. other experimental groups. TAC: ${ }^{*} \mathrm{P}<0.05$, group $\mathrm{D}$ vs. other experimental groups. The bars indicate the mean \pm standard error of the mean. C, control; CL, control group treated with lycopene; D, untreated diabetic group; DI, diabetic group treated with insulin; DL, diabetic group treated with lycopene; DIL, diabetic group treated with insulin and lycopene; TAC, total antioxidant capacity; MDA, malondialdehyde activity.

green, respectively. Cell counts in the different regions of the hippocampus (CA1, CA2, CA3, CA4 and DG; Fig. 3) revealed that the mean percentage of intact cells in each of the treated groups was significantly higher than that for diabetic rats without treatment $(\mathrm{P}<0.05)$. The results of all experimental groups are shown in Table I.

TUNEL staining results. The mean percentage of TUNEL-positive cells in the diabetic group was significantly increased compared with that in the control group $(\mathrm{P}<0.05)$. By contrast, in each of the insulin, lycopene and combination-treated groups, the mean percentage of apoptotic cells was significantly lower than that in diabetic rats without treatment $(\mathrm{P}<0.05$; Figs. 4 and 5$)$.

\section{Discussion}

The findings of the present study firstly verified that STZ induced neuronal cell death in the hippocampus region. Furthermore, diabetic animals treated with lycopene and insulin for 8 weeks exhibited attenuated STZ-induced learning and memory impairments, associated with increased TAC and decreased MDA.

STZ-induced diabetic hyperglycemia serves an important role in the degeneration of neurons in various regions of the brain, including the cingulate cortex, thalamic nuclei and hippocampus, by increasing the production of ROS (28). Oxidative stress is now recognized as an important etiological factor associated with the development of several chronic diseases, including cancer, cardiovascular 
Table I. Effects of insulin and lycopene on hippocampal neuronal cell viability (acridine orange staining).

\begin{tabular}{lcccccc}
\hline & \multicolumn{5}{c}{ Group cell death rate, $\%$} \\
\cline { 2 - 6 } Region & $\mathrm{C}$ & $\mathrm{CL}$ & $\mathrm{D}$ & $\mathrm{DI}$ & DL & DIL \\
\hline CA1 & $0.36 \pm 0.31^{\mathrm{a}}$ & $0.42 \pm 0.19$ & $18.90 \pm 1.75$ & $2.91 \pm 0.65^{\mathrm{a}}$ & $2.73 \pm 0.45^{\mathrm{a}}$ & $2.36 \pm 0.52^{\mathrm{a}}$ \\
CA2 & $0.30 \pm 0.34^{\mathrm{a}}$ & $0.34 \pm 0.26$ & $21.50 \pm 2.16$ & $2.74 \pm 0.65^{\mathrm{a}}$ & $2.58 \pm 0.63^{\mathrm{a}}$ & $2.51 \pm 0.43^{\mathrm{a}}$ \\
CA3 & $0.33 \pm 0.27^{\mathrm{a}}$ & $0.38 \pm 0.25$ & $19.00 \pm 1.70$ & $2.65 \pm 0.65^{\mathrm{a}}$ & $2.84 \pm 0.68^{\mathrm{a}}$ & $2.10 \pm 0.58^{\mathrm{a}}$ \\
CA3 & $0.46 \pm 0.32^{\mathrm{a}}$ & $0.56 \pm 0.31$ & $20.57 \pm 1.84$ & $2.60 \pm 0.92^{\mathrm{a}}$ & $2.48 \pm 0.41^{\mathrm{a}}$ & $2.36 \pm 0.73^{\mathrm{a}}$ \\
Dentate gyrus & $0.45 \pm 0.46^{\mathrm{a}}$ & $0.61 \pm 0.23$ & $18.43 \pm 1.50$ & $2.30 \pm 0.95^{\mathrm{a}}$ & $2.80 \pm 0.70^{\mathrm{a}}$ & $2.23 \pm 0.65^{\mathrm{a}}$ \\
\hline
\end{tabular}

Data are expressed as the mean \pm standard error of the mean. ${ }^{\mathrm{a}} \mathrm{P}<0.05$ vs. D group for each region. C, control; CL, control group treated with lycopene; D, untreated diabetic group; DI, diabetic group treated with insulin; DL, diabetic group treated with lycopene; DIL, diabetic group treated with insulin and lycopene.

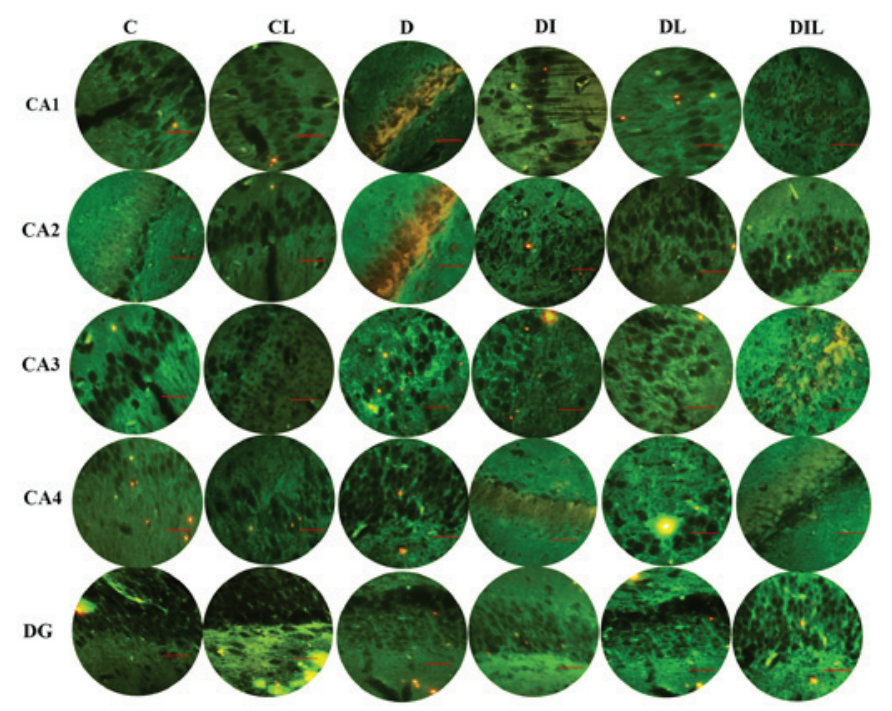

Figure 3. Effect of lycopene and insulin on cell viability in the different regions (CA1, CA2, CA3, CA4 and DG) of the hippocampus according to acridine orange staining. Magnification, $\mathrm{x} 400$; scale bar, $200 \mu \mathrm{m}$. C, control; $\mathrm{CL}$, control group treated with lycopene; D, untreated diabetic group; DI, diabetic group treated with insulin; DL, diabetic group treated with lycopene DIL, diabetic group treated with insulin and lycopene; DG, dentate gyrus.

diseases, osteoporosis and diabetes (5). Nishikawa et al (29) reported that direct glucose toxicity to neurons may be due in particular to enhanced intracellular glucose oxidation, which leads to an increase in ROS production. According to Arvanitakis et al (30), oxidative stress is a major factor influencing the extent of neuronal damage in both experimental diabetic rats and humans. Lycopene treatment may reduce the enzyme activities of superoxide dismutase, glutathione peroxidase and catalase to control levels of ROS in cadmium-treated rats, and may attenuate oxidative stress by reducing xanthine oxidase activity (31). Akbaraly et al (32) observed that lycopene enhanced cognitive performance in the elderly. Furthermore, a study by Kuhad et al (21) demonstrated that lycopene attenuated diabetes-associated cognitive decline in rats, at the levels of oxidative-nitrosative stress and peripheral inflammation associated with the development of cognitive impairment, which suggested the therapeutic potential of lycopene in

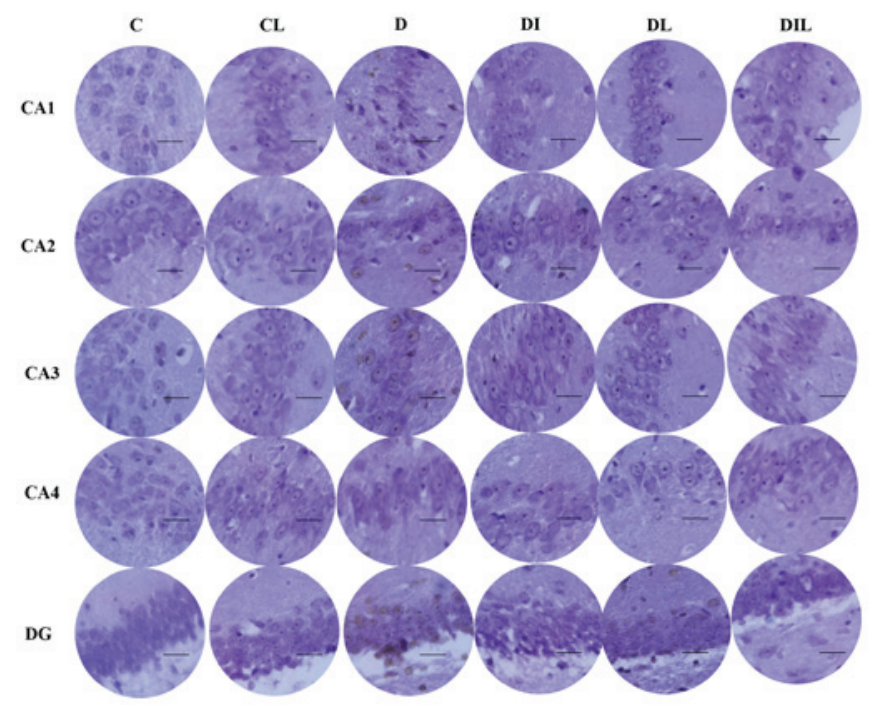

Figure 4. Effect of lycopene and insulin on cell apoptosis in the different regions (CA1, CA2, CA3 and CA4 and DG) of the hippocampus. Apoptosis was evaluated by terminal deoxynucleotidyl transferase dUTP nick-end labeling assay. Magnification, x400; scale bar, $200 \mu \mathrm{m}$. C, control; CL, control group treated with lycopene; D, untreated diabetic group; DI, diabetic group treated with insulin; DL, diabetic group treated with lycopene; DIL, diabetic group treated with insulin and lycopene; DG, dentate gyrus.

diabetes-induced learning and memory impairment. Thus, lycopene may protect against diabetes-associated memory decline through reducing oxidative stress, as evident in rat models.

The TUNEL assay results indicated that lycopene and insulin individually and simultaneously prevented apoptosis. In support of this result, Lim et al (33) reported that lycopene inhibited apoptosis in neural cells via reducing oxidative stress. They also concluded that the consumption of lycopene-rich foods may prevent neuronal damage induced by oxidative stress in certain neuropathological conditions including Alzheimer's disease. Sadek et al (34) demonstrated that lycopene could protect brain tissue from apoptotic cell death through inhibiting monosodium glutamate formation. Feng et al (35) also revealed that lycopene was a potent neuroprotectant against apoptosis during oxidative stress and mitochondrial dysfunction in the hippocampal neuroglia. 
A
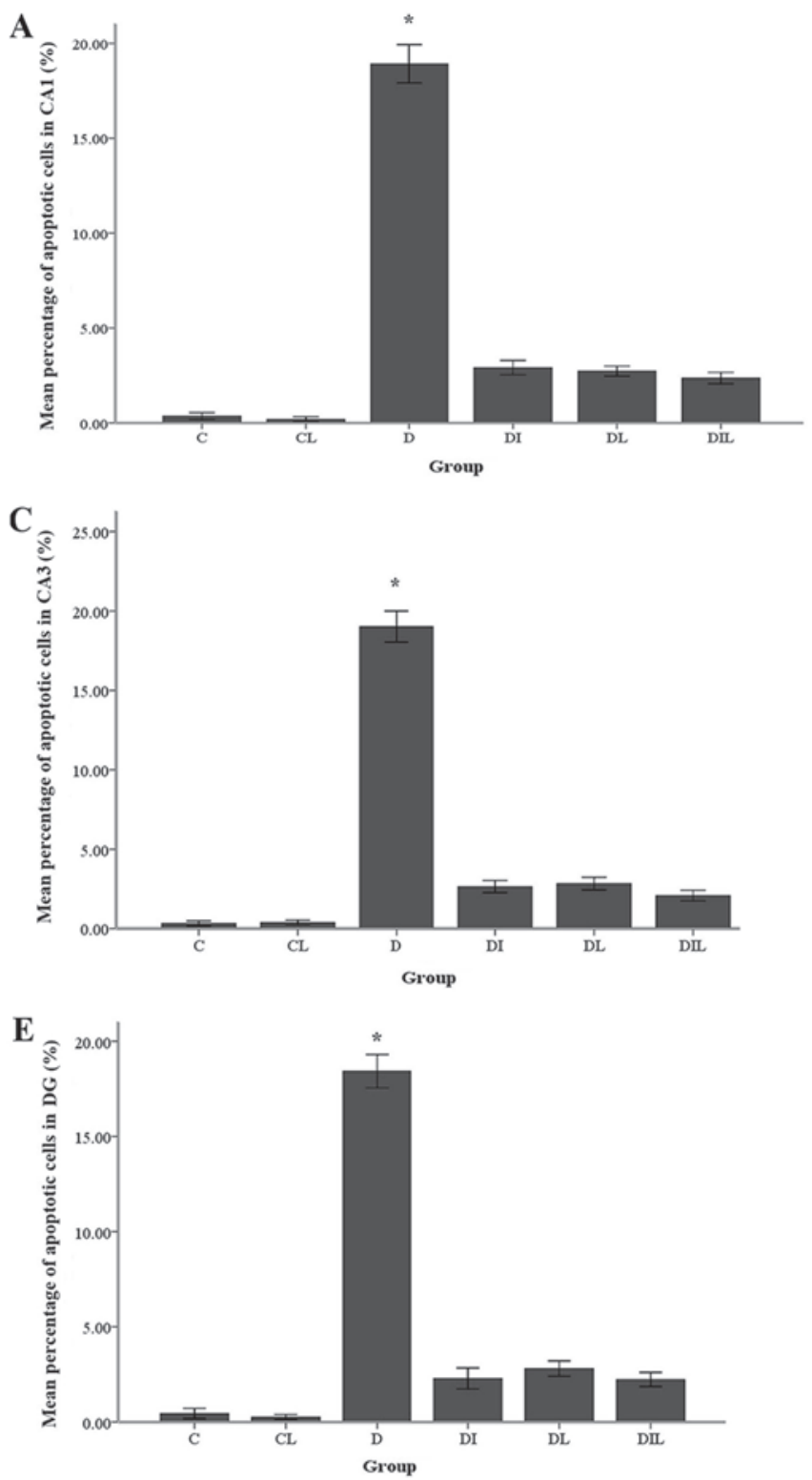

B

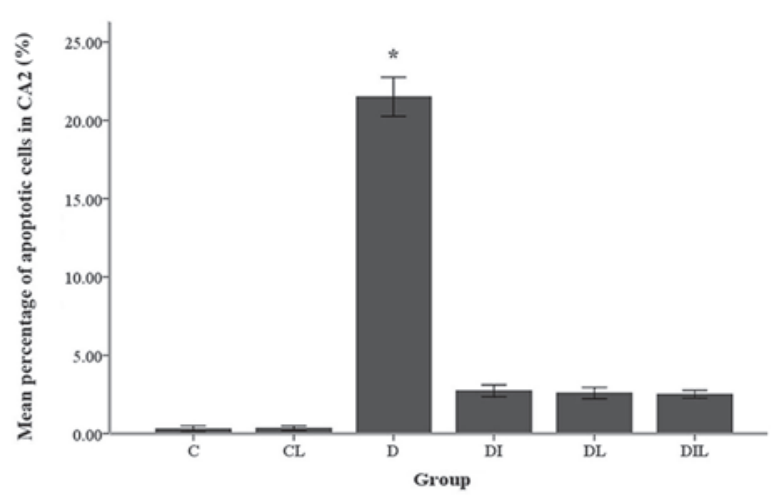

D

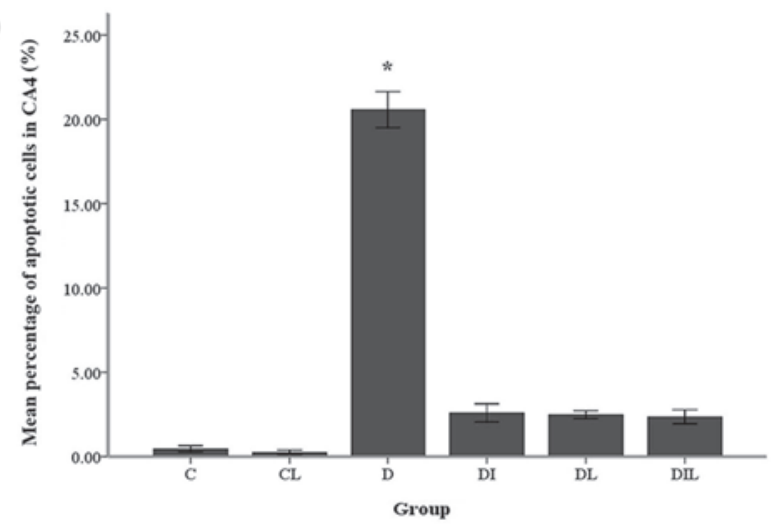

Figure 5. Effects of lycopene and insulin on the apoptosis rate of neuronal cells in the (A) CA1, (B) CA2, (C) CA3, (D) CA4 and (E) DG regions of the hippocampus. The bars indicate the mean \pm standard error of the mean. ${ }^{*} \mathrm{P}<0.05$, group $\mathrm{D}$ vs. other experimental groups. DG, dentate gyrus.

Previous study by our group demonstrated that lycopene and insulin, alone or in combination, could prevent apoptosis in the hippocampus region of STZ-induced diabetic rats, with increased expression of anti-apoptotic genes (Bcl-2 and $\mathrm{Bcl}-\mathrm{xL}$ ) and decreased expression of pro-apoptotic gene Bax (36). Data obtained presently indicated decreased TAC and increased MDA in the hippocampi of diabetic rats. Plasma TAC represents an appropriate biochemical parameter for comparing the overall antioxidant status. Lipid peroxidation is a well-established mechanism associated with cellular injury and is frequently used as an indicator of oxidative stress (37). It has previously been revealed that in STZ-induced diabetic rats, TCA, MDA and antioxidative enzymes including catalase are altered not only in plasma, but also in other organs (including the kidney, liver, heart and brain) (1). The current results indicated that lipid peroxidation levels were significantly increased, while glutathione, superoxide dismutase and catalase activities were markedly decreased in the hippocampus of diabetic rats. It has been shown that treatment with lycopene may recover the levels of lipid peroxides, glutathione, superoxide dismutase and catalase to control values (38). Witztum (39) reported that lipid peroxidation products increased oxidative stress conditions and ROS. Consistent with the present study, Zhang et al (40) and Prakash and Kumar (41) reported that dietary antioxidants, including lycopene, could protect important biomolecules and DNA from oxidation and serve an important role in the prevention of chronic diseases such as Alzheimer's and Parkinson's disease.

In conclusion, the current data suggested that insulin and lycopene are effective in protecting hippocampal neuroglia against STZ-induced damage leading to learning and memory impairment. Therefore, insulin and lycopene, alone or in combination, may be considered as drug candidates for neurological disorders in which oxidative stress serves an important role in pathogenesis. 


\section{Acknowledgements}

The authors would like to thank Dr Alireza Shoghli, Deputy of Research and Technology of Zanjan University of Medical Sciences, Zanjan, Iran, for assisting with the research.

\section{Funding}

The present study was part of an anatomy graduate student research thesis, which was funded by the Deputy of Research and Technology at the Zanjan University of Medical Sciences, Zanjan, Iran with code (grant no. A-12-202-3).

\section{Availability of data and materials}

All primary data generated in the present study are archived at the Zanjan University of Medical Sciences, Zanjan, Iran, and available from the corresponding author on request.

\section{Authors' contributions}

RM performed the experiments. AA acted as study advisor and helped to revise the manuscript. DS acted as the study advisor. IJA is the corresponding author, analyzed data, supervised the project, and wrote the manuscript. All authors discussed the results and contributed to the final manuscript.

\section{Ethics approval and consent to participate}

The animal experimental protocols were reviewed and approved by the Institutional Animal Ethics Committee of Zanjan University of Medical Sciences, Zanjan, Iran (approval no. ZUMS.REC.1394.29).

\section{Patient consent for publication}

Not applicable.

\section{Competing interests}

The authors declare that they have no competing interests.

\section{References}

1. Tian X, Liu Y, Ren G, Yin L, Liang X, Geng T, Dang H and An R: Resveratrol limits diabetes-associated cognitive decline in rats by preventing oxidative stress and inflammation and modulating hippocampal structural synaptic plasticity. Brain Res 1650: 1-9, 2016.

2. Rahmeier FL, Zavalhia LS, Tortorelli LS, Huf F, Géa LP, Meurer RT, Machado AC, Gomez R and Fernandes MDC: The effect of taurine and enriched environment on behaviour memory and hippocampus of diabetic rats. Neurosci Lett 630: $84-92,2016$

3. Esteghamati A,Larijani B, Aghajani MH, Ghaemi F, Kermanchi J, Shahrami A, Saadat M, Esfahani EN, Ganji M, Noshad S, et al: Diabetes in Iran: Prospective Analysis from First Nationwide Diabetes Report of National Program for Prevention and Control of Diabetes (NPPCD-2016). Sci Rep 7: 13461, 2017.

4. Diaz-Valencia PA, Bougnères P and Valleron AJ: Global epidemiology of type 1 diabetes in young adults and adults: A systematic review. BMC Public Health 15: 255, 2015.

5. Koppula S, Kumar H, More SV, Kim BW, Kim IS and Choi DK: Recent advances on the neuroprotective potential of antioxidants in experimental models of Parkinson's disease. Int J Mol Sci 13: 10608-10629, 2012.
6. Martín-Gallán P, Carrascosa A, Gussinyé M and Domínguez C: Biomarkers of diabetes-associated oxidative stress and antioxidant status in young diabetic patients with or without subclinical complications. Free Radic Biol Med 34: 1563-1574, 2003.

7. Wolff SP, Jiang ZY and Hunt JV: Protein glycation and oxidative stress in diabetes mellitus and ageing. Free Radic Biol Med 10: 339-352, 1991.

8. Baynes JW: Role of oxidative stress in development of complications in diabetes. Diabetes 40: 405-412, 1991.

9. Fukui K, Onodera K, Shinkai T, Suzuki S and Urano S: Impairment of learning and memory in rats caused by oxidative stress and aging, and changes in antioxidative defense systems. Ann N Y Acad Sci 928: 168-175, 2001.

10. Reagan LP: Glucose, Stress, and Hippocampal Neuronal Vulnerability. Int Rev Neurobiol 51: 289-324, 2002.

11. Reagan LP: Insulin signaling effects on memory and mood. Curr Opin Pharmacol 7: 633-637, 2007.

12. Zilliox LA, Chadrasekaran K, Kwan JY and Russell JW: Diabetes and Cognitive Impairment. Curr Diab Rep 16: 87, 2016.

13. Gaspar JM, Baptista FI, Galvão J, Castilho AF, Cunha RA and Ambrósio AF: Diabetes differentially affects the content of exocytotic proteins in hippocampal and retinal nerve terminals. Neuroscience 169: 1589-1600, 2010

14. Anjum R and Khar A: Apoptosis: An overview. Indian J Biotechnol 1: 58-72, 2002.

15. Sun J, Chu Y-F, Wu X and Liu RH: Antioxidant and antiproliferative activities of common fruits. J Agric Food Chem 50: 7449-7454, 2002

16. Klipstein-Grobusch K, Launer LJ, Geleijnse JM, Boeing H, Hofman A and Witteman JC: Serum carotenoids and atherosclerosis. The Rotterdam Study. Atherosclerosis 148: 49-56, 2000.

17. Miller NJ, Sampson J, Candeias LP, Bramley PM and Rice-Evans CA: Antioxidant activities of carotenes and xanthophylls. FEBS Lett 384: 240-242, 1996.

18. Rao AV and Rao LG: Carotenoids and human health. Pharmacol Res 55: 207-216, 2007.

19. Jafari Anarkooli I, Barzegar Ganji H and Pourheidar M: The protective effects of insulin and natural honey against hippocampal cell death in streptozotocin-induced diabetic rats. J Diabetes Res 2014: 491571, 2014.

20. Özkan Y, Yilmaz O, Oztürk Aİ and Erşan Y: Effects of triple antioxidant combination (vitamin $\mathrm{E}$, vitamin $\mathrm{C}$ and $\alpha$-lipoic acid) with insulin on lipid and cholesterol levels and fatty acid composition of brain tissue in experimental diabetic and non-diabetic rats. Cell Biol Int 29: 754-760, 2005.

21. Kuhad A, Sethi R and Chopra K: Lycopene attenuates diabetes-associated cognitive decline in rats. Life Sci 83: $128-134,2008$

22. Firouzjaei MA, Jafari MR, Eskandari M, Anarkoli IJ and Alipour M: Aminoguanidine changes hippocampal expression of apoptosis-related genes, improves passive avoidance learning and memory in streptozotocin-induced diabetic rats. Cell Mol Neurobiol 34: 343-350, 2014.

23. Khaki A, Novin MG, Khaki AA, Nouri M, Sanati E and Nikmanesh M: Comparative study of the effects of gentamicin, neomycin, streptomycin and ofloxacin antibiotics on sperm parameters and testis apoptosis in rats. Pak J Biol Sci 11: 1683-1689, 2008

24. Alipour M, Gholami MR, Jafari Anarkooli I, Sohrabi D, Tajki J and Pourheidar M: Intraperitoneal aminoguanidine improves sciatic nerve ischemia-reperfusion injury in male sprague-dawley rats. Cell Mol Neurobiol 31: 765-773, 2011.

25. Prieto P, Pineda M and Aguilar M: Spectrophotometric quantitation of antioxidant capacity through the formation of a phosphomolybdenum complex: Specific application to the determination of vitamin E. Anal Biochem 269: 337-341, 1999.

26. Wills ED: Mechanisms of lipid peroxide formation in animal tissues. Biochem J 99: 667-676, 1966.

27. Wokes F and Still BM: The estimation of protein by the biuret and Greenberg methods. Biochem J 36: 797-806, 1942.

28. Li ZG and Sima AA: C-peptide and central nervous system complications in diabetes. Exp Diabesity Res 5: 79-90, 2004.

29. Nishikawa T, Edelstein D, Du XL, Yamagishi S, Matsumura T, Kaneda Y, Yorek MA, Beebe D, Oates PJ, Hammes HP, et al: Normalizing mitochondrial superoxide production blocks three pathways of hyperglycaemic damage. Nature 404: 787-790, 2000.

30. Arvanitakis Z, Wilson RS, Bienias JL, Evans DA and Bennett DA: Diabetes mellitus and risk of Alzheimer disease and decline in cognitive function. Arch Neurol 61: 661-666, 2004. 
31. Rencuzogullari N and Erdogan S: Oral administration of lycopene reverses cadmium-suppressed body weight loss and lipid peroxidation in rats. Biol Trace Elem Res 118: 175-183, 2007.

32. Akbaraly NT, Faure H, Gourlet V, Favier A and Berr C: Plasma carotenoid levels and cognitive performance in an elderly population: Results of the EVA Study. J Gerontol A Biol Sci Med Sci 62: 308-316, 2007.

33. Lim S, Hwang S, Yu JH, Lim JW and Kim H: Lycopene inhibits regulator of calcineurin 1-mediated apoptosis by reducing oxidative stress and down-regulating Nucling in neuronal cells. Mol Nutr Food Res 61: 1600530, 2017.

34. Sadek K, Abouzed T and Nasr S: Lycopene modulates cholinergic dysfunction, Bcl-2/Bax balance, and antioxidant enzymes gene transcripts in monosodium glutamate (E621) induced neurotoxicity in a rat model. Can J Physiol Pharmacol 94: 394-401, 2016.

35. Feng C, Luo T, Zhang S, Liu K, Zhang Y, Luo Y and Ge P: Lycopene protects human SH SY5Y neuroblastoma cells against hydrogen peroxide induced death via inhibition of oxidative stress and mitochondria associated apoptotic pathways. Mol Med Rep 13: 4205-4214, 2016.
36. Soleymaninejad M, Joursaraei SG, Feizi F and Jafari Anarkooli I: The Effects of lycopene and insulin on histological changes and the expression level of Bcl-2 family genes in the hippocampus of streptozotocin-induced diabetic rats. J Diabetes Res 2017: 4650939, 2017.

37. Moore K and Roberts LJ II: Measurement of lipid peroxidation. Free Radic Res 28: 659-671, 1998.

38. Sharma S, Anjaneyulu M, Kulkarni SK and Chopra K: Resveratrol, a polyphenolic phytoalexin, attenuates diabetic nephropathy in rats. Pharmacology 76: 69-75, 2006.

39. Witztum JL: The oxidation hypothesis of atherosclerosis. Lancet 344: 793-795, 1994.

40. Zhang YJ, Gan RY, Li S, Zhou Y, Li AN, Xu DP and Li HB: Antioxidant phytochemicals for the prevention and treatment of chronic diseases. Molecules 20: 21138-21156, 2015.

41. Prakash A and Kumar A: Lycopene protects against memory impairment and mito-oxidative damage induced by colchicine in rats: An evidence of nitric oxide signaling. Eur J Pharmacol 721: 373-381, 2013. 